\title{
A Case Report of Proximal Migration of Fibular Head with Intact Peroneal Nerve in a Post-Operative Infected Tibia Gap Non-union Managed by Ilizarov Technique
}

\author{
Spandan R Koshire ${ }^{1}$, Rajesh R Koshire ${ }^{2}$, Ajay M Wankhade ${ }^{1}$
}

Learning Point of the Article:

Management of infected tibia gap non-union with Ilizarov technique requires meticulous and regular post-operative monitoring and patient education to detect any fibular complications which may arise during the osteogenesis distraction period.

\section{Abstract}

Introduction: We hereby present a rare case of proximal fibular head migration associated with Ilizarov technique for infected tibia gap nonunion due to follow-up failure in post-operative management during the coronavirus disease (COVID) pandemic.

Case Report: A 45-year-old male patient had undergone primary external fixation with wound debridement for a compound tibia shaft fracture Grade 3 A Gustilo class which later on with a healed external wound with a discharging sinus at the fracture site was confirmed to be infected nonunion and subsequently managed by Ilizarov ring fixation over an antibiotic coated intramedullary nail and local gentamycin beads after a necrotic bone fragment of around $6 \mathrm{~cm}$ was excised. Post-operative protocol of compression and distraction was initiated and the patient educated regarding the same before discharge. As the world over was hit by the COVID pandemic and the lockdown limited all possible movements in our country, the patient could not follow up for monitoring the Ilizarov limb lengthening procedure. He presented to us after 4 months after the relaxation of lockdown with radiological union at the docking site but with a shortening of about $3 \mathrm{~cm}$. Vigorous knee range physiotherapy failed to improve range beyond $90^{\circ}$ which prompted us to check X-ray the knee joint and revealed the complication of proximal fibular head migration of $4 \mathrm{~cm}$ but with no neurodeficit and currently the patient is being managed with full weight mobilization with the help of crutch and shoe raise and an improved knee range till $100^{\circ}$ of flexion with no pain tenderness or any other complaints.

Conclusion: Having knowledge of this possible rare complication and the need for follow-up and monitoring with the importance of patient education makes practicing orthopedic surgeons equipped to handle and anticipate such undesirable complications.

Keywords: Bony union, fibula migration, fibula resection, Ilizarov technique, infected non-union.

\section{Introduction}

In recent times, there has been an increasing trend of long bones fractures of compound nature due to high-energy trauma. The most common one fractured is tibia due its susceptible subcutaneous location. Among the commonly acquired complications in such a case are delayed union and nonunion. Tibia is predisposed to non-union more often than any other bone in the body [1].

In addition, many other factors complicate the union process such as long-term infection, loss of bone and soft tissue, and limb length discrepancy [2]. Infected tibial non-union is an anticipated challenge for orthopedic surgeons. The management options for chronic long-standing diaphyseal infections associated with non-union include extensive debridement with rotational flaps, defect filling with antibiotic cement beads most commonly containing gentamycin [3], Papineau-type open cancellous bone grafting, free microvascular soft-tissue and bone transplants, tibiofibular synostosis, and the Ilizarov method [4]. The Ilizarov method

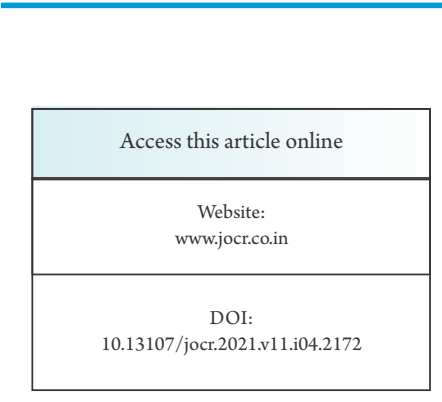

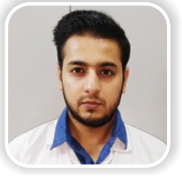

Dr. Spandan R Koshire

Author's Photo Gallery

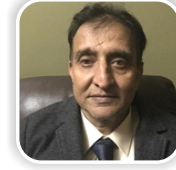

Dr. Rajesh R Koshire

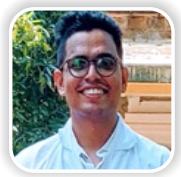

Dr. Ajay M Wankhade
Department of Orthopaedics, Seth Gordhandas Sunderdas Medical College and King Edward Memorial Hospital, Mumbai, Maharashtra, India, ${ }^{2}$ Department of Orthopaedic and Health and Family Welfare, District Civil HOSPITAL, Nasik, Maharashtra, India.

Address for Correspondence:

Dr. Spandan R Koshire,

Department of Orthopaedics, Seth Gordhandas Sunderdas Medical College and King Edward Memorial Hospital,

Mumbai, Maharashtra, India.

E-mail: spndnkoshire11@gmail.com

Journal of Orthopaedic Case Reports | pISSN 2250-0685 | eISSN 2321-3817 | Available on www.jocr.co.in | doi:10.13107/jocr.2021.v11.i04.2172 This is an Open Access article distributed under the terms of the Creative Commons Attribution Non-Commercial License (http://creativecommons.org/licenses/by-nc/3.0) which permits unrestricted non-commercial use, distribution, and reproduction in any medium, provided the original work is properly cited. 


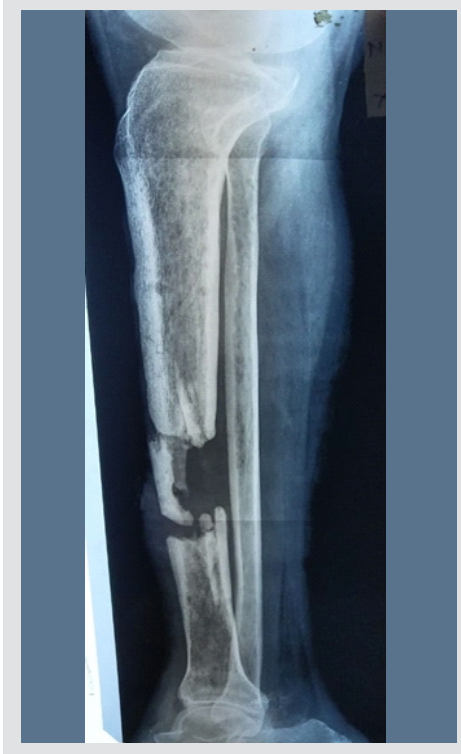

Figure 1: Lateral X-ray of the right leg showing necrotic and osteomyelitic bone fragment.

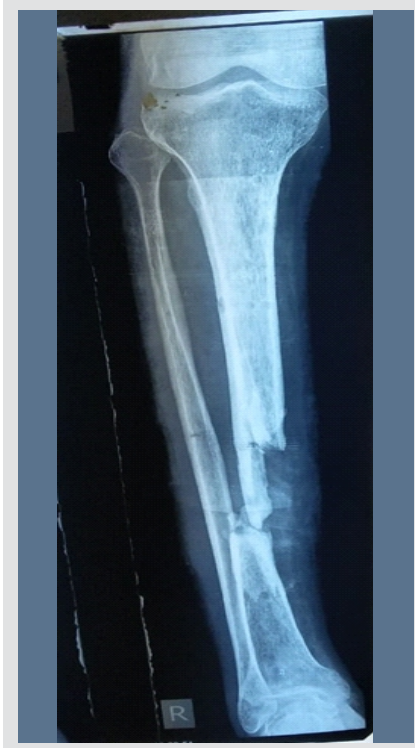

Figure 2: Anteroposterior X-ray of the right leg showing necrotic and osteomyelitic bone fragment.

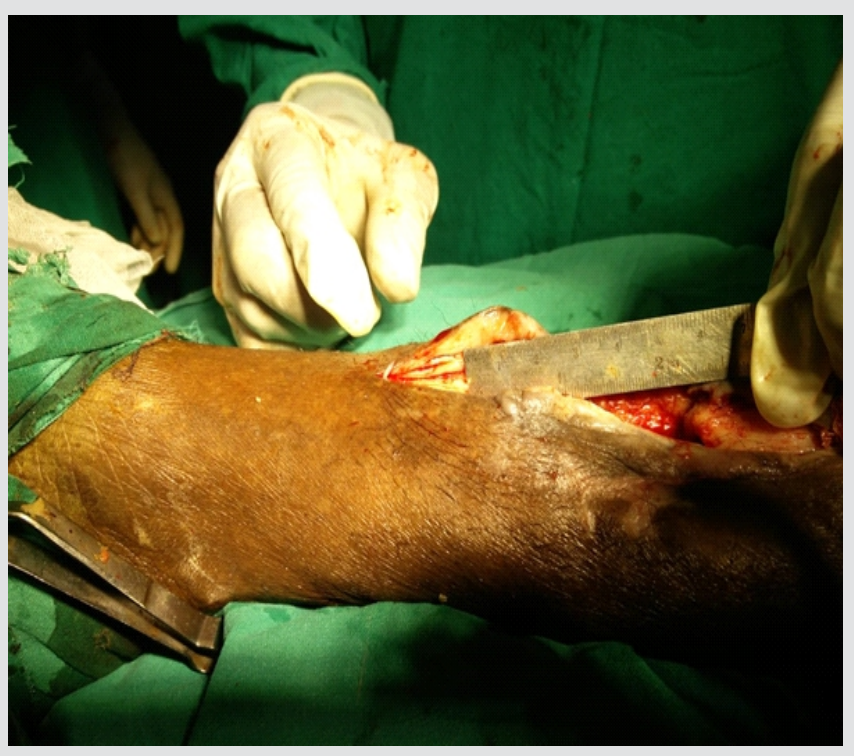

Figure 3: Clinical intraoperative image showing bone gap of $6 \mathrm{~cm}$.

\section{Case Report}

Mr. CD, a 45-year-old manual laborer, with no history of any medical illness, non-smoker, alleged motor vehicle accident (pedestrian vs. car) 11/2 years back and sustained right-sided open comminuted fracture distal third tibia Gustilo type $3 \mathrm{~A}$. The initial wound debridement and external fixation were done on day 0 of accident to manage the bony and soft-tissue damage. During the initial procedure, we carried out tests for the antibiotic sensitivity and the organism identification, the resultant organism being Staphylococcus aureus and the sensitive antibiotics were initiated and continued for around 6 weeks, post-trauma, the wound was healed up but the fracture was still undergoing union and only minimal callus formation was present. Diagnosed with osteomyelitis, the patient was taken up for right-sided tibia debridement, sequestrectomy, and resection of necrotic bone fragment (Fig. 1, 2). $6 \mathrm{~cm}$ bone fragment was resected with oscillating saw at the distal tibial developing country like India where the patient education and general level of understanding are poor.

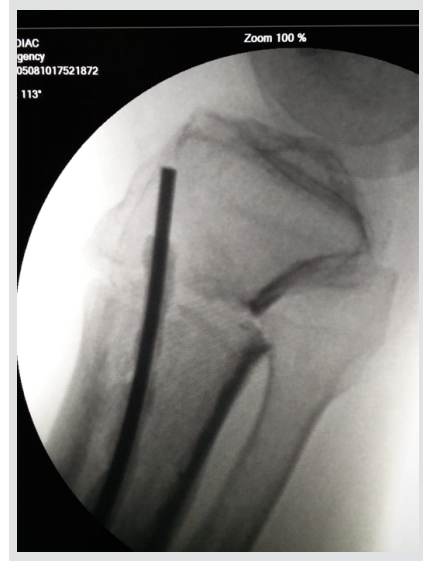

Figure 4: Proximal corticotomy site with in situ intramedullary nail.

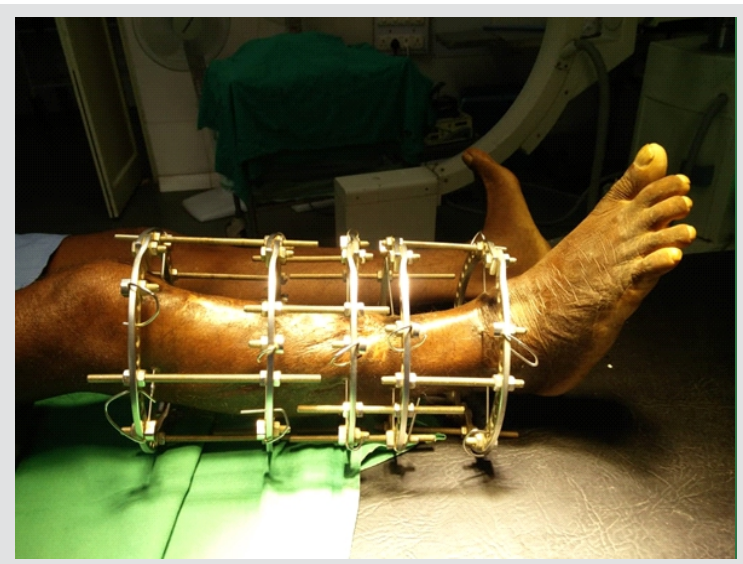

Figure 5: Proximal corticotomy site with in situ intramedullary nail.

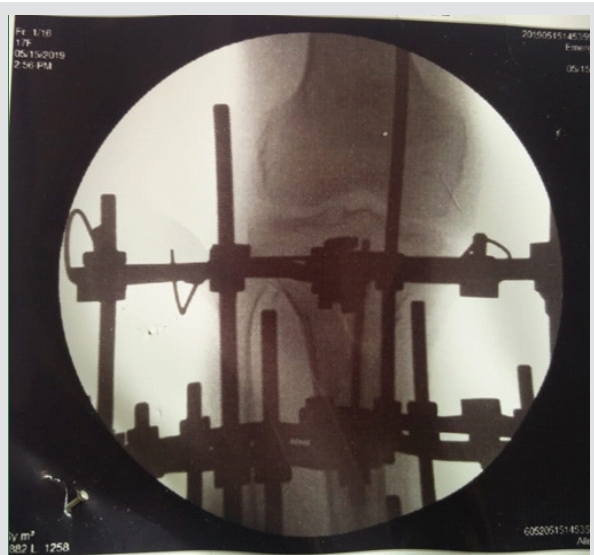

Figure 6: X-ray image of initiation of osteogenesis distraction sequence. 


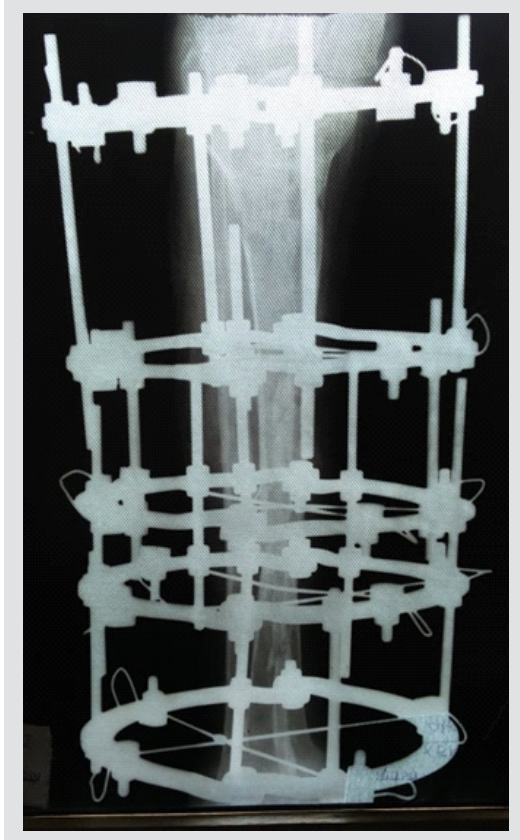

Figure 7: Follow-up X-ray showing complete bony union at the docking site.

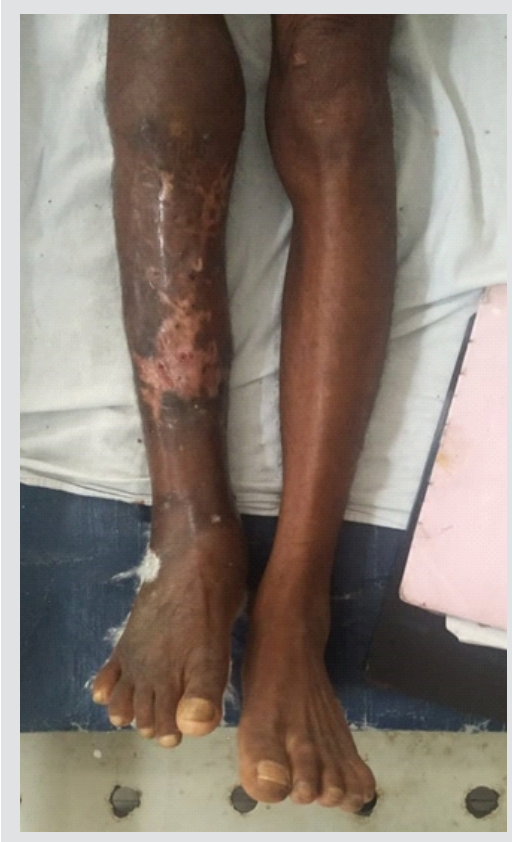

Figure 8: Clinical image showing limb shortening on the affected right side.

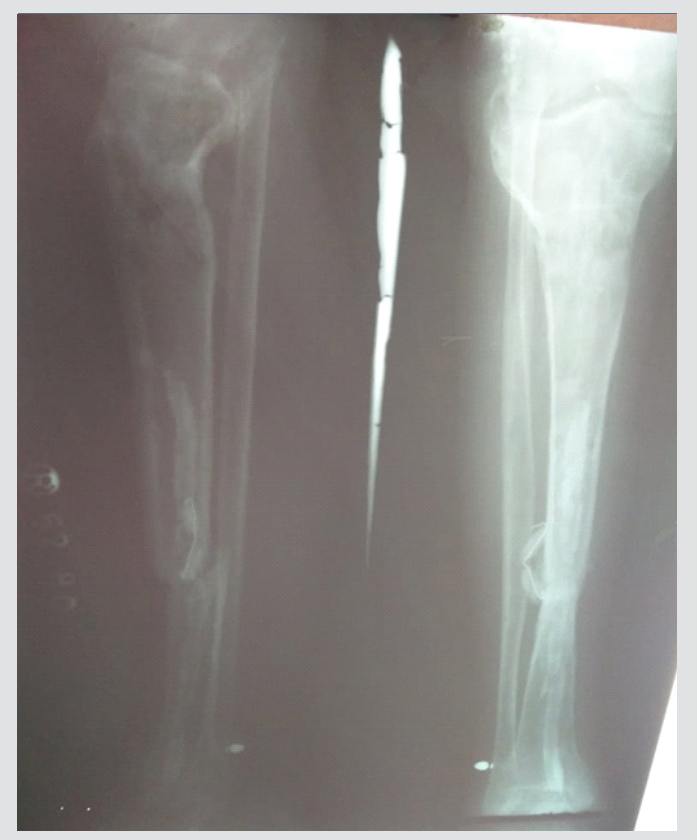

Figure 9: Anterior to posterior and lateral X-ray image of the right leg with knee showing proximal fibular head migration above the level of the kne joint. shaft site, up to the presumed healthy area during the operation (Fig. 3). After resection of necrotic bone fragment it was followed by proximal tibial metaphyseal corticotomy after passing an antibiotic coated intramedullary nail with local gentamycin beads insertion (Fig. 4). Four days later, Ilizarov ring fixator procedure was done over the antibiotic coated intramedullary nail (Fig. 5). Gradual compression and docking was planned after the 8 th day of ring fixation as the bone loss at the initial measurement after debridement was $6 \mathrm{~cm}$ (Fig. 6). At the outset of the pandemic, the patient was discharged and instructed to follow up for check X-rays and monitoring for the compression distraction process. The patient himself made the distraction and compression regulations but failed to follow up during the lockdown period. When he returned back to us for follow-up, checkX-ray was done (Fig. 7).

It revealed union at the fracture docking site. At this point, kindly note that check X-ray failed to reveal the proximal fibular head migration due to the rings obstructing the radiographic field also the knee flexion was limited to $90^{\circ}$ due to hindrance by the rings. Further, as bony union was established, we proceeded with exchange of Ilizarov apparatus with an above knee cast and walking heel for mobilization. At this time, we also noted a limb length discrepancy of about $3 \mathrm{~cm}$ (Fig. 8).

After $1 \frac{1}{2}$ months, the cast was removed fracture site tenderness, mobility was absent and knee range of motion was initiated, even after continuous physiotherapy range was not improving beyond $90^{\circ}$ flexion which prompted us to X-ray image the knee. The radiograph revealed proximal fibular migration of $4 \mathrm{~cm}$ (Fig. 9).

On further examination, we confirmed peroneal nerve function to be intact. The current status of the patient is painless ambulation with the help of a crutch with no neurodeficit with length discrepancy of $3 \mathrm{~cm}$ with a shoe raise and with a knee range from $0^{\circ}$ to $100^{\circ}$ planned for a proximal fibulectomy and removal of gentamycin beads at a later date.

\section{Discussion}

Ilizarov technique has been a time tested procedure for bone defect management since the past due to its ability to provide a strong mechanical framework, provision for wound management and also allows weight-bearing, but it also has a fair share of complications related to the relatively long and time-consuming nature [6]. Ilizarov technique has provided us with a salvage option to preserve the limb against the inevitable amputation [7]. Limb lengthening with the help of a combined Ilizarov ring fixator and an intramedullary nail has proven efficacy [8]. It has been a documented fact that proximal and distal migration of fibula at the malleolar end is an anticipated complication along with valgus deformity at the knee as well as ankle [6]. In our case, fibular complication arose but in terms of proximal fibular head migration at the knee joint. This procedure apart from the intricate and tedious intraoperative course also requires a much longer and patient approach in the post-operative period during the osteogenesis and distraction. The patient needs to be well informed about the apparatus nature and the necessity for regular monitoring with follow-up [9]. In our case, the failure to follow up due to the coronavirus 
disease pandemic led to unregulated post-operative compression distraction and the resultant fibular complication.

\section{Conclusion}

For infected tibia non-union which has already undergone stabilization procedure initially for primary wound and fracture management, it can be best managed by Ilizarov external fixator over an implanted antibiotic coated intramedullary nail as it can provide bone transport defect management along with wound care and offers an opportunity to salvage the limb to avoid the inevitable amputation. But of utmost importance in this case scenario which we would like to highlight to our readers is the need for follow-up and monitoring of the patient during the post-operative period. We recommend, therefore, that the patient should be educated regarding the compression and distraction sequence at home along with the need for follow-up for check radiographs to monitor the status of the docking site, corticotomy site, and the knee joint as in our case, we encountered a rare complication of proximal fibular head migration of $4 \mathrm{~cm}$ with intact common peroneal nerve function.

\section{Clinical Message}

Management of infected tibia gap non-union with Ilizarov technique is a time tested and a successful treatment modality but also the one which requires meticulous and regular postoperative monitoring and patient education to detect any fibular complications which may arise during the osteogenesis distraction period.

\section{References}

1. Fahad S, Habib AA, Awais MB, Umer M, Rashid HU. Infected non-union of tibia treated with ilizarov external fixator: Our experience. Malays OrthopJ 2019;13:36-41.

2. Chaudhary MM. Infected nonunion of tibia. Indian J Orthop 2017;51:256-68.

3. Inzana JA, Schwarz EM, Kates SL, Awad HA. Biomaterials approaches to treating implant-associated osteomyelitis. Biomaterials 2016;81:58-71.

4. Dendrinos GK, Kontos S, Lyritsis E. Use of the Ilizarov technique for treatment of non-union of the tibia associated with infection. J Bone Joint Surg Am 1995;77:835-46.

5. Yin P,Ji Q, Li T, LiJ, LiZ, Liu J, et al. A systematic review and meta-analysis of ilizarov methods in the treatment of infected nonunion of tibia and femur. PLoS One
2015; 10:e0141973.

6. Kim SJ, Agashe MV, Song SH, Song HR. Fibula-related complications during bilateral tibial lengthening: 60 patients followed for mean 5 years. Acta Orthop 2012;83:271-5.

7. Saleh M, Bashir HM, Farhan MJ, McAndrew AR, Street R. Tibial lengthening: Does the fibula migrate. J Pediatr Orthop B 2002;11:302-6.

8. Gulabi D, Erdem M, Cecen GS, Avci CC, Saglam N, Saglam F. Ilizarov fixator combined with an intramedullary nail for tibial nonunions with bone loss: Is it effective. Clin Orthop Relat Res 2014;472:3892-901.

9. Ilizarov GA. Clinical application of the tension-stress effect for limb lengthening. Clin Orthop Relat Res 1990;250:826.
Conflict of Interest: Nil

Source of Support: Nil

Consent: The authors confirm that informed consent was obtained from the patient for publication of this case report

\section{How to Cite this Article}

Koshire SR, Koshire RR, Wankhade AM. A Case Report of Proximal Migration of Fibular Head with Intact Peroneal Nerve in a PostOperative Infected Tibia Gap Non-union Managed by Ilizarov Technique. Journal of Orthopaedic Case Reports 2021 April;11(4): 104-107. 